\title{
Loss of breast cancer metastasis suppressor 1 promotes ovarian cancer cell metastasis by increasing chemokine receptor 4 expression
}

\author{
XIU-JIE SHENG ${ }^{1}$, YING-QUN ZHOU ${ }^{1}$, QING-YUAN SONG ${ }^{1}$, DONG-MEI ZHOU ${ }^{1}$ and QI-CAI LIU ${ }^{2}$ \\ ${ }^{1}$ Department of Obstetrics and Gynecology, The Third Affiliated Hospital of Guangzhou Medical College, Guangzhou 510150; \\ ${ }^{2}$ Experimental Medical Research Center of Guangzhou Medical College, Guangzhou 510182, P.R. China
}

Received November 1, 2011; Accepted December 2, 2011

DOI: 10.3892/or.2011.1596

\begin{abstract}
Breast cancer metastasis suppressor 1 (BRMS1) is a predominantly nuclear protein that differentially regulates the expression of multiple genes, leading to suppression of metastasis without affecting orthotopic tumor growth. It has been demonstrated that BRMS1 may be correlated with advanced ovarian cancer. The aim of this study was to investigate the mechanisms of BRMS1 involvement in ovarian cancer metastasis. We constructed a plasmid containing a short hairpin RNA (shRNA) against BRMS1 and transfected it into the ovarian cancer cell line OVCAR3. Real-time reverse transcription polymerase chain reaction (real-time PCR) and Western blot analyses demonstrated that BRMS1 expression was efficiently downregulated. Stable suppression of BRMS1 significantly enhanced cell adhesion, migration, invasion and angiogenesis. We also found that chemokine receptor 4 (CXCR4) was upregulated at both the mRNA and protein levels. When approaching for the mechanism, we discovered that activation of the nuclear factor $-\kappa \mathrm{B}(\mathrm{NF}-\kappa \mathrm{B})$ signaling pathway mediated CXCR4 upregulation, as demonstrated by the electrophoretic mobility shift assay (EMSA). Collectively, these results suggest that attenuation of BRMS1 may play a critical role in promoting migration, invasion and angiogenesis of ovarian cancer cells and BRMS1 may regulate the metastatic potential at least in part through upregulation of CXCR4 via NF- $\kappa \mathrm{B}$ activation. Restoration of BRMS1 function is thus a potential new strategy for treating human ovarian cancer.
\end{abstract}

\section{Introduction}

Ovarian carcinoma is the leading cause of death from gynecological malignancies. The majority of ovarian cancer

Correspondence to: Dr Xiu-Jie Sheng, Department of Obstetrics and Gynecology, The Third Affiliated Hospital of Guangzhou Medical College, Guangzhou 510150, P.R. China

E-mail: xjsheng@gzemail.cn

Key words: ovarian neoplasm, breast cancer metastasis suppressor 1, RNA interference, metastasis, chemokine receptor 4 patient deaths are due to the irreversible physiological effects of metastases on normal organ function rather than from the primary tumor (1). Despite recently improved chemotherapeutic agents and an increased 5-year survival rate, ovarian cancer mortality remains unchanged. A better understanding of the metastatic mechanisms of ovarian cancer is therefore needed to determine effective therapeutic interventions to either eradicate or slow metastatic outgrowth.

Many factors are involved in regulating metastasis through diverse mechanisms. Among metastasis suppressors, breast cancer metastasis suppressor 1 (BRMS1) was originally shown to functionally suppress the metastatic capacities of breast cancer cells (2). Further studies showed that BRMS1 is not only a metastasis suppressor gene in breast cancer models but also in various other cancers, such as melanoma and ovarian cancer $(3,4)$. Zhang et al (4) demonstrated that low levels of BRMS1 expression correlated with poor prognosis in ovarian cancer patients. They further showed that transfection of BRMS1 complementary DNA (cDNA) into the highly malignant ovarian carcinoma cell line HO-8910PM significantly reduced cell adhesion, motility and invasion in vitro and also decreased the incidence of lung metastasis without affecting tumor growth. BRMS1 is thought to regulate metastasis through multiple mechanisms, including restoration of gap junctions, reduction of phosphoinositide signaling, interaction with the histone deacetylase complex and regulation of the nuclear factor $-\kappa \mathrm{B}(\mathrm{NF}-\kappa \mathrm{B})$ pathway (5-7). In particular, several metastasis-related genes were reported to be downregulated by BRMS1 through modulating the activity of $\mathrm{NF}-\kappa \mathrm{B}$, including osteopontin (OPN), urokinase-type plasminogen activator (uPA), microRNA-146, interleukin-6 (IL-6) and chemokine receptor 4 (CXCR4) (8-12).

Chemokines are small cytokines that are characterized by their capacity to induce directional cellular migration towards a gradient of chemokines by binding to chemokine receptors. One of the most extensively studied chemokine receptors is CXCR4, which selectively binds the chemokine stromal cellderived factor-1 (SDF-1) also known as CXCL12 (13). Recent evidence suggests that the SDF-1/CXCR4 pathway is involved in local invasion and metastasis of many cancers, including breast cancer, gastric cancer and ovarian cancer (14-16). Not only that, CXCR4 has been observed to promote angiogenesis 
by stimulating the secretion of several angiogenic factors, such as vascular endothelial growth factor and IL-6 $(17,18)$. Interestingly, a recent study by Yang et al demonstrated that BRMS1 reduces CXCR4 expression in lung cancer cells via abrogation of NF- $\kappa \mathrm{B}$ activation (12); however, the functional implications of BRMS1 and its relationship to the CXCR4 signaling pathway in ovarian neoplasms are not clear.

Therefore, we investigated the potential mechanisms of BRMS1-mediated metastasis suppression in ovarian cancer. In this study, recombinant plasmid containing short-hairpin RNA (shRNA) sequences targeting BRMS1 mRNA transcription regions was constructed and transfected into ovarian cancer cells. Their influences on cell adhesion, migration, invasion and angiogenesis were observed, and the expression of CXCR4 was detected. Finally, we employed an electrophoretic mobility shift assay (EMSA) to explore whether BRMS1 regulates CXCR4 expression through the NF- $\kappa \mathrm{B}$ pathway. Our data indicate that BRMS1 negatively regulates metastatic potential at least in part through the suppression of NF- $\kappa \mathrm{B}$-dependent CXCR4 expression.

\section{Materials and methods}

Cell lines and cell culture. The human ovarian cancer cell line OVCAR3 (ATCC, USA) was grown in Dulbecco's modified Eagle's medium (DMEM) (Gibco, Invitrogen, USA) supplemented with $10 \%$ fetal bovine serum (FBS) (Gibco, Invitrogen) and antibiotics (100 U/ml penicillin and $100 \mu \mathrm{g} / \mathrm{ml}$ streptomycin). Human umbilical venous endothelial cells (HUVECs) were obtained from the Institute of Biochemistry and Cell Biology of the Chinese Academy of Science (Shanghai) and cultured in Kaighn's modified Ham's F-12K medium (Mediatech, Manassas, VA, USA) supplemented with endothelial cell growth supplement (BD Biosciences, Canada) and 10\% FBS. Cultures were tested and shown free of mycoplasma contamination. All cells were maintained in $5 \% \mathrm{CO}_{2}$ atmosphere at $37^{\circ} \mathrm{C}$. For all functional and biological assays, cells with $>95 \%$ viability were used at $70-90 \%$ confluence.

Plasmids construction. Based on the preliminary results of screening out effective silencing siRNA sequences, the following double-stranded RNA oligonucleotides specific for the BRMS1 coding region were used: 5'-CACCGTTCGTACTT ATTCCTGATCACATCCTTCAAGAGAGGATGTGATCAG GAATAAGTACGAATTTTTTG-3' (sense), 5'-GATCCAA AAAATTCGTACTTATTCCTGATCACATCCTCTCTTG AAGGATGTGATCAGGAATAAGTACGAAC-3' (antisense); Negative control sequences with no significant homology to the BRMS1 gene and which had the sequence not present in the human, mouse or rat genome databases were: 5'-CACCGT TCTCCGAACGTGTCACGTCAAGAGATTACGTGACACG TTCGGAGAATTTTTTG-3' (sense), 5'-GATCCAAAAAAT TCTCCGAACGTGTCACGTAATCTCTTGACGTGACACG TTCGGAGAAC-3' (antisense). All DNA chains were synthesized by GenePharma Co. (Shanghai, China). The plasmids were extracted and the accuracy of the constructs was confirmed by DNA sequencing.

Cell transfection. According to the manufacturer's protocol for Lipofectamine 2000 (Invitrogen), pGPU6/GFP/Neo-BRMS1 or pGPU6/GFP/Neo-NC were transfected into OVCAR3 cells. After $6 \mathrm{~h}$, the cultures were replaced with $2 \mathrm{ml}$ fresh medium supplemented with $10 \%$ FBS and antibiotics. Then the cells were visualized under fluorescence microscopy. After $48 \mathrm{~h}$, $600 \mu \mathrm{g} / \mathrm{ml} \mathrm{G} 418$ (Sigma, USA) was added to the medium for selecting stable transfectants, and individual clones were isolated and maintained in a medium containing $300 \mu \mathrm{g} / \mathrm{ml}$ G418. Real-time PCR and Western blotting were applied to analyze BRMS1 mRNA and protein levels, respectively. The stably transfected OVCAR3 cells were named BRMS1shRNA (transfected with pGPU6/GFP/Neo-BRM-S1) and NC-shRNA, respectively.

Real-time reverse transcription polymerase chain reaction (real-time PCR). Total RNA from cells was extracted with TRIzol Reagent (Invitrogen) following the manufacturer's instruction. cDNA was synthesized from total RNA using the PrimeScript RT reagent kit (Takara, Japan). The cDNA specimens were amplified using the SYBR Premix Ex Taq ${ }^{\mathrm{TM}}$ (Takara). GAPDH gene was used as an internal control for standardization in triplicate. Cycle conditions were: $95^{\circ} \mathrm{C}$ for $30 \mathrm{sec}$, followed by 40 cycles of $95^{\circ} \mathrm{C}$ for $5 \mathrm{sec}, 60^{\circ} \mathrm{C}$ for $34 \mathrm{sec}$ and finally $95^{\circ} \mathrm{C}$ for $15 \mathrm{sec}, 60^{\circ} \mathrm{C}$ for $1 \mathrm{~min}$. PCR amplification was performed on the ABI 7500 Sequence Detection System (PE Applied Biosystems, Foster City, CA, USA). The comparative $\mathrm{Ct}(\Delta \Delta \mathrm{CT})$ method was used to determine the expression fold change. The sequences of the primers used were as follows: BRMS1 forward: 5'-ATGCCTGTCCAGCCTCC AAG-3' and reverse 5'-GCGTCGCTCATAGTCCTCATCA-3'; CXCR4 forward: 5'-GGTGGTCTATGTTGGCGTCT-3' and reverse 5'-CTCAGTGGAAACAGATGAAT-3'; GAPDH forward: 5'-GCACCGTCAAGGCTGAGAAC-3' and reverse 5'-TGGT GAAGACGCCAGTGGA-3'.

Western blot analysis. Total cellular proteins from the cells were obtained using RIPA lysis buffer (Santa Cruz Biotechnology, USA) containing a cocktail of proteinase inhibitors and phosphatase inhibitors. Protein concentrations were measured using the BCA protein assay (Sigma). The proteins were subjected to $10 \%$ SDS denatured polyacrylamide gel and transferred onto PVDF membranes. Membranes were blocked in 5\% non-fat milk for $1 \mathrm{~h}$ at $4^{\circ} \mathrm{C}$ and blotted with rabbit anti-human antibody at the recommended dilution BRMS1 (1:500, BioWorld, USA), CXCR4 (1:100, Epitomics, USA) and $\beta$-actin (1:500, BioWorld), and subsequently incubated with the appropriate secondary antibody. After washing with TBST, visualization of the second antibody was performed using a chemiluminescence detection procedure according to the manufacturer's protocol (Amersham Biosciences, Japan). The LabWorks ${ }^{\mathrm{TM}}$ Image Acquisition and Analysis Software (UVP, USA) was used to quantify band intensities. $\beta$-actin was used as a loading control.

Cell adhesion assay. For this assay, 96-well plates were incubated with $50 \mu \mathrm{l}(30 \mu \mathrm{g} / \mathrm{ml}) \mathrm{BD}$ Matrigel $^{\mathrm{TM}}$ Matrix (BD Biosciences, Germany) at $4^{\circ} \mathrm{C}$ overnight, then washed with PBS twice and blocked with $1 \%$ BSA for $1 \mathrm{~h}$ at $37^{\circ} \mathrm{C}$. Cells were trypsinized and seeded at $1 \times 10^{5} / \mathrm{ml}$ to each coated well and incubated for $2 \mathrm{~h}$ in $5 \% \mathrm{CO}_{2}$ atmosphere at $37^{\circ} \mathrm{C}$, then rinsed three times with PBS to remove non-adherent cells. Each well with $100 \mu \mathrm{l}$ medium was added $20 \mu \mathrm{l}$ 
A

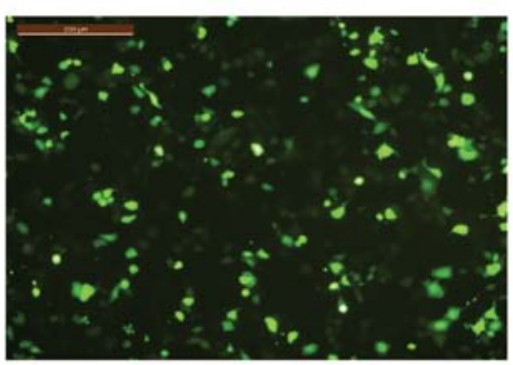

C

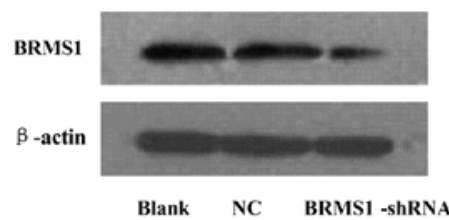

B
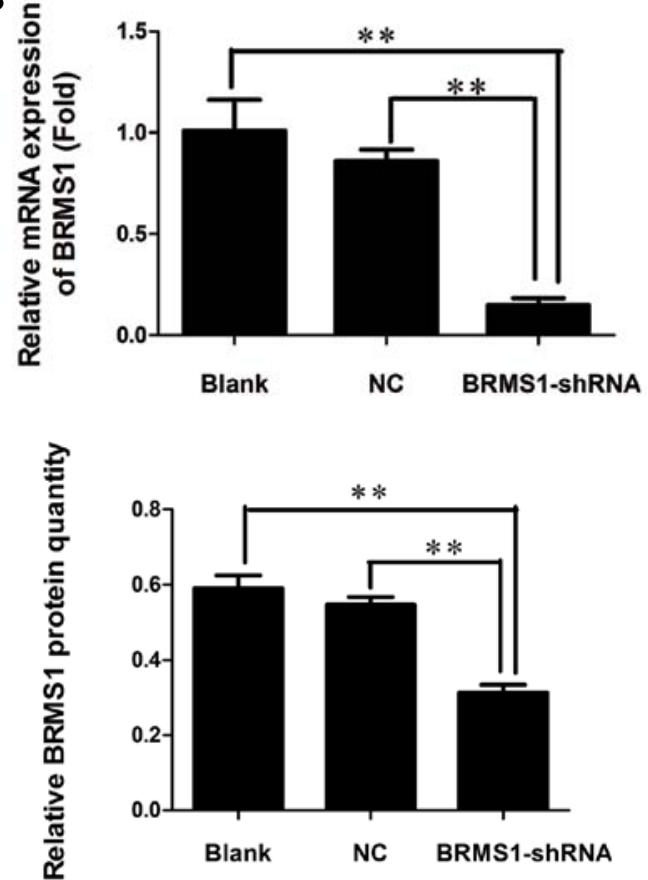

Figure 1. Suppression of BRMS1 expression by small hairpin RNA (shRNA) in OVCAR3 cells. (A) Representative pictures of OVCAR3 cells 24 h after transfection with BRMS1-shRNA (x100 magnification). (B) The relative expression of BRMS1 mRNA was analyzed by real-time PCR. (C) Total protein was extracted and measured by Western blot analysis. $\beta$-actin was used as a loading control. Both an untransfected control (blank control) and a non-specific sequence control (NC) were also included. Data shown are the means $\pm \mathrm{SD}$ of a representative experiment performed in triplicate $\left({ }^{* *} \mathrm{P}<0.01\right)$.

3-(4,5-dimethylthiazol-2-yl)-2,5-diphenyltetrazolium bromide (MTT), further incubated for $4 \mathrm{~h}$, then the MTT was removed and $150 \mu \mathrm{l}$ dimethylsulfoxide (DMSO) was pipetted into each well. The optical density (OD) was measured at $570 \mathrm{~nm}$ with a microplate reader. The OD values were propotional to the number of cells with adhesion; five duplicate wells were set up for each group.

Migration assay. Cell migration was assessed by adding $5 \times 10^{4}$ cells into the upper chamber of an $8-\mu \mathrm{m}$ pore size Transwell insert (Corning, USA) in serum-free media. These inserts were placed in wells with serum-containing media. After seeding for $24 \mathrm{~h}$, non-migrating cells were removed from the upper surface of the filter with a cotton-tipped swab. The cells on the lower surface of the filter were fixed in $4 \%$ paraformaldehyde and stained using crystal violet staining solution. Five random fields were counted at x100 magnification. All the data presented are from at least three independent experiments performed in duplicate.

Matrigel invasion assay. Transwell inserts ( $8-\mu \mathrm{m}$ pore size) coated with $30 \mu \mathrm{l}$ Matrigel were placed in wells as previously described. In the top chamber, $5 \times 10^{4}$ cells were plated in serum-free media and incubated with serum-containing media as a chemoattractant in the bottom chamber. Cells were then incubated at $37^{\circ} \mathrm{C}$ and allowed to invade through the Matrigel barrier for $24 \mathrm{~h}$. After incubation, non-invading cells were removed using a cotton swab. Filters were fixed and stained with crystal violet staining solution, and five random fields were counted at x100 magnification. All the data presented are from at least three independent experiments performed in duplicate.
In vitro tube formation assay. OVCAR3 cells were cultured in 6-well plates with fresh complete medium for $24 \mathrm{~h}$ and $1 \mathrm{ml}$ conditioned medium was collected. For tube formation assay, the 48-well plates were coated with Matrigel (100 $\mu \mathrm{l}$ per well) and kept in $5 \% \mathrm{CO}_{2}$ atmosphere at $37^{\circ} \mathrm{C}$ for $30 \mathrm{~min}$. Then, $5 \times 10^{4}$ HUVECs were suspended in $500 \mu \mathrm{l}$ conditioned medium and applied to the pre-coated 48 -well plates. After incubation at $37^{\circ} \mathrm{C}$ for another $24 \mathrm{~h}$, images were captured under a microscope and the tubular structures formed in the Matrigel were counted at x100 magnification in five random fields.

Electrophoretic mobility shift assay (EMSA). The assay is based on that DNA-protein complexes migrate slower than unbound DNA double-stranded oligonucleotides on a native polyacrylamide gel, resulting in a 'shift' in the migration of the labeled DNA band. The detection of bands was performed by the LightShift ${ }^{\mathrm{TM}}$ Chemiluminescent EMSA kit (Pierce, USA) that used a non-isotopic method to detect DNA-protein interactions. Nuclear extracts were prepared from OVCAR3 cells knockdown of BRMS1 and the control sample. Nuclear proteins were incubated at room temperature for $10 \mathrm{~min}$ with oligonucleotide probe bearing an NF- $\mathrm{NB}$ binding sequence on the CXCR4 promoter (5'-TCCCCTGGGCTTCCCAAGCC-3'). The probe was labeled with a biotin at its 5'-end. Another oligonucleotide with the same sequence but without labeling was used as a competitive sequence at 500 -fold concentration. After the reaction the DNA-protein complexes were subjected to a $6.5 \%$ native polyacrylamide gel electrophoresis and transferred to a nylon membrane. Then the membrane was immediately cross-linked for $15 \mathrm{~min}$ on a UV transilluminator equipped with $312 \mathrm{~nm}$ bulbs. Finally, a chemiluminescent detection method utilizing a luminal/enhancer solution and a 


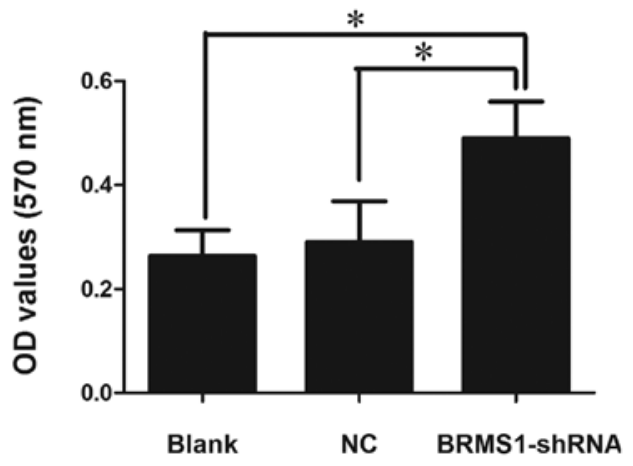

Figure 2. Knockdown of BRMS1 expression enhances the adhesion of OVCAR 3 cells. Adhesion was examined by determining the optical densities (OD) at $570 \mathrm{~nm}$. OVCAR3 cells stably expressing BRMS1-shRNA exhibited a significant increase in adhesion. Results are expressed as the average optical densities (means $\pm \mathrm{SD},{ }^{*} \mathrm{P}<0.05$ ).

stable peroxide solution was used as described by the manufacturer and membranes were exposed to X-ray films for 2-5 min before developing.

Statistical analysis. All experiments were performed at least in triplicate and data were compiled from three separate experiments. The results were calculated as means \pm SD. All statistical analyses were determined by one-way ANOVA using the SPSS16.0 software. A P-value $<0.05$ was considered significant.

\section{Results}

Specific inhibition of BRMS1 expression by BRMS1-shRNA. Plamid vectors expressing either BRMS1-shRNA or nonspecific sequence control (NC) shRNA were constructed and transfected into OVCAR3 cells. After $24 \mathrm{~h}$, high transfection efficiency was observed by fluorescence microscopy (Fig. 1A). To determine silencing efficiency, the expression levels of BRMS1 mRNA and protein were measured by real-time PCR and Western blot analysis, respectively. The BRMS1 mRNA level declined significantly in the BRMS1-shRNA transfected cells, with an average inhibition of $85.15 \%$ compared to the blank control group $(\mathrm{P}<0.01$, Fig. 1B). BRMS1 protein expression was also decreased, with an average inhibition of $46.67 \%$ in the BRMS1-shRNA group ( $\mathrm{P}<0.01$, Fig. 1C). These results suggested that pGPU6/GFP/Neo-BRMS1 could effectively suppress BRMS1 expression at both the mRNA and protein levels in OVCAR3 cells.

Effect of BRMS1-shRNA on adhesion. Cancer cell adhesion to the subendothelial extracellular matrix is an important step in metastasis formation. To assess the potential involvement of downregulation of BRMS1 expression on adhesion, a cell adhesion assay was employed. Our results showed that BRMS1shRNA cells markedly enhanced cell adhesion to the Matrigel matrix. The OD values at $570 \mathrm{~nm}$ were proportionate to the number of attached cells. Cell adhesion of BRMS1-shRNA

A

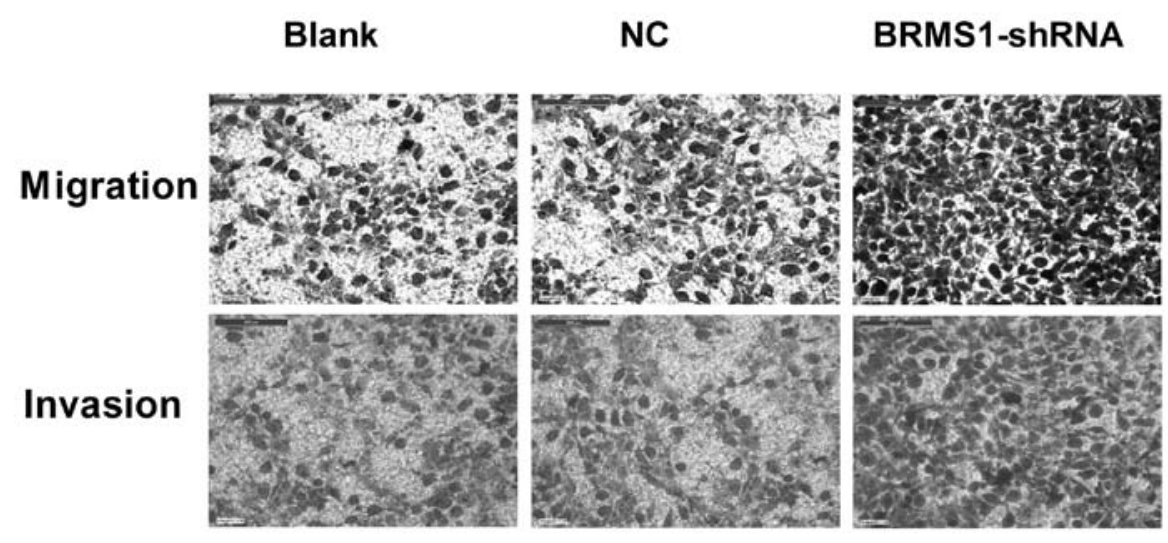

$\mathbf{B}$
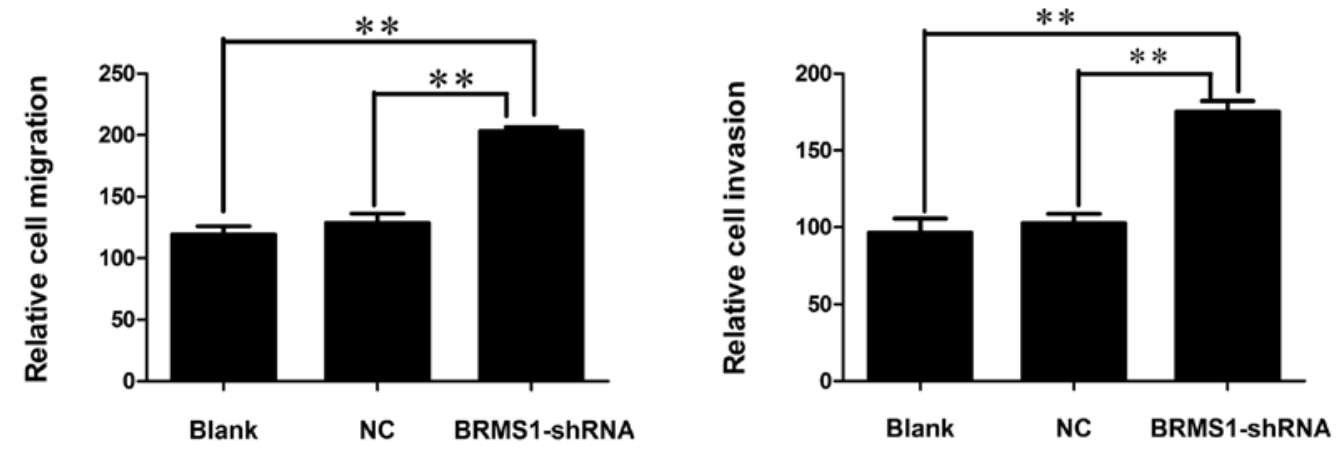

Figure 3. Inhibition of BRMS1 expression promotes migration and invasion of OVCAR3 cells. (A) Representative crystal violet staining of migrated and invaded cells. (B) The average migrated and invaded cell numbers of OVCAR3 cells transfected with BRMS1-shRNA were significantly higher than those of both the negative and blank control groups (migration: 203.33 $\pm 5.68,128.67 \pm 13.43$ and $119.33 \pm 11.59$; invasion: $175.33 \pm 11.72,102.67 \pm 10.41$ and $96.67 \pm 15.63$ ) Data are represented as the means \pm SD of three independent experiments $\left({ }^{* *} \mathrm{P}<0.01\right)$. 
A

\author{
Blank
}

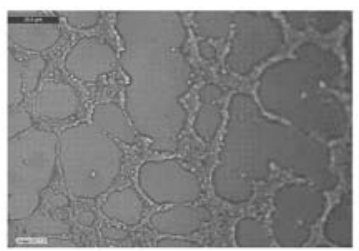

B

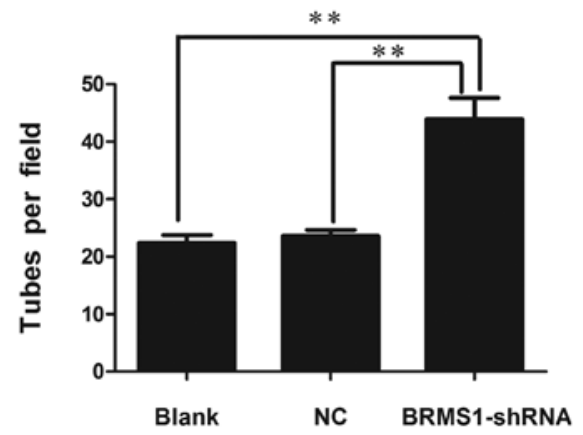

NC

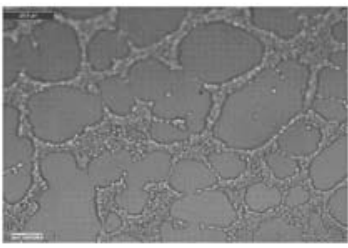

BRMS1-ShRNA

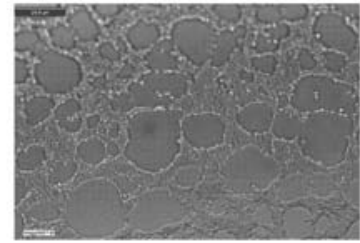

Figure 4. BRMS1 silencing induces ovarian cancer cells angiogenesis. (A) Capillary-like tubes were observed and photographed. BRMS1 knockdown in ovarian cancer cells promoted HUVECs tube formation. (B) The numbers of tubes formed per field were counted in 5 random fields for the BRMS1 knockdown, negative control and blank control groups (43.93 \pm 6.33 , $23.60 \pm 1.78$ and $22.33 \pm 2.52$ ). Data are presented as means \pm SD from three independent experiments ( $\left.{ }^{* *} \mathrm{P}<0.01\right)$. transfected OVCAR 3 cells was increased by 1.88 -fold compared to the blank control cells (Fig. 2).

Effect of BRMS1-shRNA on migration and invasion. Migration and Matrigel invasion assay were performed to examine the impact of BRMS1-shRNA on cell migration and invasion, respectively. As shown in Fig. 3, BRMS1 knockdown increased OVCAR3 cell migration by 1.7 -fold compared to untreated cells. The Matrigel invasion assay results demonstrated that the invasiveness of cells treated with BRMS1-shRNA increased by 1.81-fold compared to the control group. Taken together, these data indicated that BRMS1-shRNA promoted motility and invasion of OVCAR3 cells.

Effect of BRMS1-shRNA on angiogenesis. Angiogenesis plays a critical role in the growth and metastatic potential of all solid tumors. To determine the effects of BRMS1 silencing on ovarian cancer cell angiogenesis, we utilized the tube formation assay. Compared to the corresponding control, the average number of complete tubular structures formed by HUVECs was increased by 1.97 -fold in conditioned medium in OVCAR3 cells transfected with BRMS1-shRNA. These data indicated that inhibition of BRMS1 significantly enhanced the angiogenic capacity of the ovarian cancer cells (Fig. 4).

Upregulation of CXCR4 by BRMSI-shRNA. Recently, BRMS1 was shown to regulate metastatic potential through the downregulation of CXCR4 (12). To determine whether loss of BRMS1 regulated CXCR4 expression in ovarian cancer, CXCR4 mRNA and protein levels of OVCAR3 cells transfected with either BRMS1-shRNA or a negative control were measured by real-time RT-PCR and Western blot analysis, respectively. As shown in Fig. 5, CXCR4 mRNA was 1.78-fold higher in BRMS1-shRNA transfected cells compared to the blank control group. Furthermore, Western blot analysis revealed that BRMS1 silencing in OVCAR3 cells elevated CXCR4 protein levels by 1.26 -fold. These results elucidated that knockdown of BRMS1 upregulated CXCR4 at both the transcriptional
A

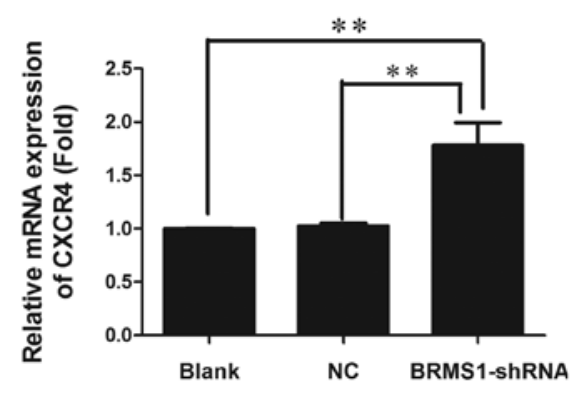

B

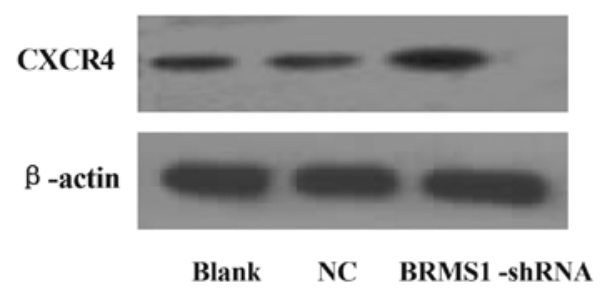

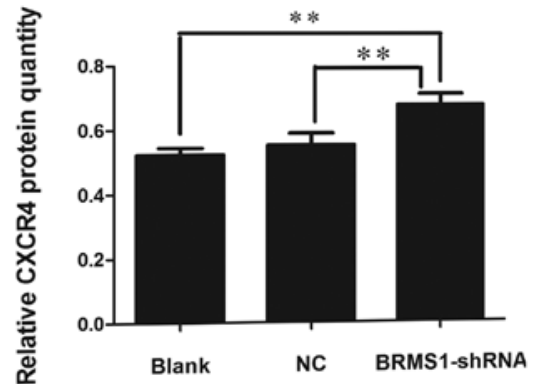

Figure 5. Suppression of BRMS1 upregulates CXCR4 at both the transcriptional and translational levels. (A) Total RNA was extracted from OVCAR3 cells transfected with either BRMS1-shRNA or NC-shRNA, and CXCR4 mRNA expression was measured by real-time RT-PCR. When BRMS1 was blocked, CXCR4 mRNA was increased compared to control cells ( $\left.{ }^{* *} \mathrm{P}<0.01\right)$. (B) Protein was extracted from OVCAR3 cells transfected with either BRMS1-shRNA or NC-shRNA and CXCR4 protein level was measured by Western blot analysis. $\beta$-actin was used as a loading control. Results revealed that BRMS1 silencing in OVCAR3 cells elevated CXCR4 protein by 1.26 -fold $\left({ }^{* *} \mathrm{P}<0.01\right)$. 


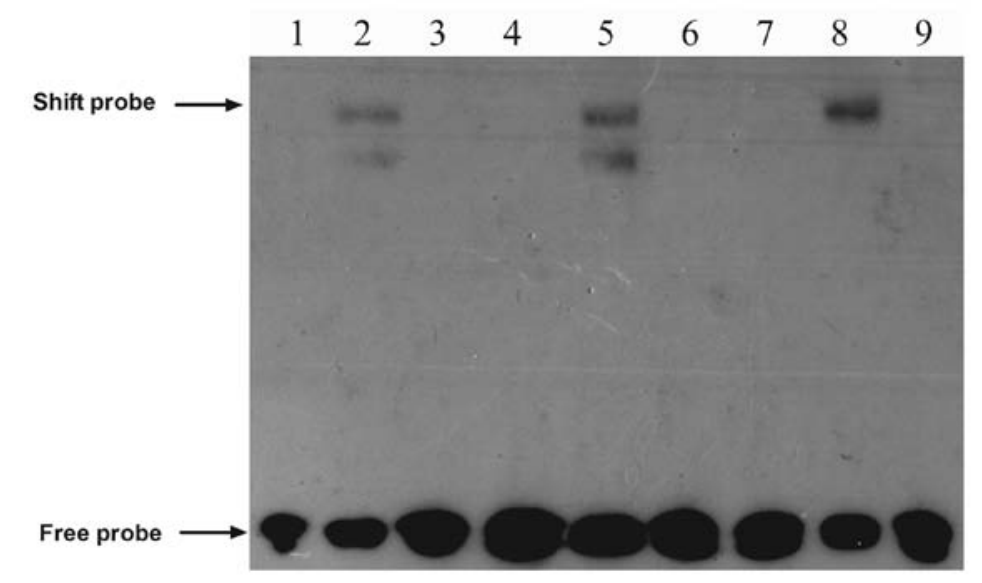

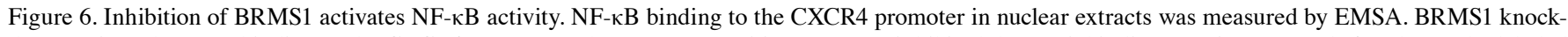
down activated NF- $\mathrm{KB}$ binding to the CXCR4 promoter, whereas a competitive sequence inhibited the DNA binding reaction. Lanes 1, 4 and 7 , probe labeled with biotin; Lane 2, probe + nuclear extract from untreated OVCAR3 cells; Lane 3, probe + competitive probe + nuclear extract from untreated OVCAR3 cells; Lane 5, probe + nuclear extract from OVCAR3 cells transfected with a non-specific sequence; Lane 6, probe + competitive probe + nuclear extract from OVCAR3 cells transfected with a non-specific sequence; Lane 8, probe + nuclear extract from OVCAR3 cells transfected with BRMS1- shRNA; Lane 9, probe + competitive probe + nuclear extract from OVCAR3 cells transfected with BRMS1-shRNA.

and translational levels. Taken together, our data indicate that loss of BRMS1 expression induces adhesion, migration, invasion and angiogenesis of OVCAR3 cells, which may be due to upregulation of CXCR4.

BRMS1 knockdown promotes CXCR4 expression through activation of the $N F-\kappa B$ signaling pathway. A prior study revealed that NF- $\mathrm{KB}$ promoted breast cancer migration and invasion by directly upregulating CXCR4 expression (19). Therefore, we chose to perform an EMSA to explore whether BRMS1 regulates CXCR4 expression through the NF- $\mathrm{kB}$ pathway in ovarian cancer cells. The EMSA results suggested that BRMS1 knockdown increased the DNA binding activity of NF- $\mathrm{kB}$ to the CXCR4 promoter, compared to the control groups, whereas the unlabeled competitive sequence markedly inhibited this binding (Fig. 6).

\section{Discussion}

Metastasis is a multistep process involving dissociation of malignant cells in the primary tumor, local invasion, angiogenesis, intravasation, survival in the circulation, extravasation and proliferation at a secondary site (20). These processes are modulated by many factors among which the metastasis suppressors are of particular importance for elucidating the underlying genetic and molecular biological mechanisms of metastasis.

The metastasis suppressor gene BRMS1 was discovered by Seraj et al (2) while studying the non-random amplifications and deletions in chromosome 11 using differential display. It is located on chromosome 11q 13.1-13.2 and consists of 10 exons and 9 introns spanning approximately $7 \mathrm{~kb}$. Previous research showed that introducing BRMS1 into the highly metastatic ovarian cancer cell line HO-8910PM significantly suppressed adhesion, motility and local invasion without affecting tumor growth in vitro (4). Moreover, a recent report suggested that BRMS1 was associated with tumor angiogenesis. Loss of BRMS1 resulted in deficient suppression of vasculogenesis and contributed to melanoma metastasis (11). BRMS1 has also been shown to reduce the capacity of multiple human cancer cell lines to metastasize to the lymph nodes, lungs and/or bone in experimental models $(2,21,22)$. In addition, BRMS1 has clinical relevance for some tumor types. BRMS1 mRNA expression was downregulated in breast tumor tissues (7) and in breast cancer brain metastases (23). Hicks et al (24) claimed that attenuation of BRMS1 expression in breast carcinomas was associated with reduced disease-free survival in the context of hormone receptor-negativity or HER 2 overexpression. Furthermore, Zhao and Wang (25) observed that BRMS1 expression in ovarian serous adenocarcinoma was significantly lower than in both normal ovarian tissue and benign ovarian tumor tissue. BRMS1 was correlated with surgical stage, lymph node metastasis and tumor size. Another study determined that both BRMS1 mRNA and protein levels were diminished in non-small cell lung cancer (NSCLC) compared to the adjacent non-cancerous lung. Preservation of BRMS1 expression was accordingly associated with improved survival of NSCLC patients (26). Recently, an increasing number of studies have demonstrated the potential of using BRMS1 as a prognostic marker and therapeutic target for breast cancer (27), ovarian cancer (4), melanoma (11) and NSCLC (26). Together, the data provide compelling evidence that BRMS1 is an effective metastasis suppressor in tumors; however, the mechanistic basis for its metastasis-suppressive function in human ovarian cancer is poorly defined.

In this study, we employed RNA interference (RNAi) technology to knock down endogenous BRMS1 expression and analyzed the influence of BRMS1 on the metastatic behavior of ovarian cancer cells. Due to the stability and long-term effectiveness of shRNA, BRMS1-shRNA was constructed and transfected into the human ovarian cancer cell line OVCAR3. Our data revealed that the expression of BRMS1 mRNA and protein was decreased in OVCAR3 cells following BRMS1shRNA transfection, with inhibition rates of $85.15 \%$ at the mRNA level and $46.67 \%$ at the protein level. We then focused on cell adhesion, migration, invasiveness and angiogenesis, all 
of which are essential steps for the establishment of metastasis. We found that BRMS1 silencing increased adhesion, migration and invasion, and induced vascularization of ovarian cancer cells. Consistent with results reported in the literature, we determined that BRMS1 is indeed an effective metastasis suppressor in ovarian cancer.

Numerous studies have confirmed that many BRMS1 downstream targets are involved in regulating tumor progression and metastatic behaviors. It has also been reported that these processes are associated with $\mathrm{NF}-\kappa \mathrm{B}$ signaling pathways. Cicek et al (7) demonstrated that BRMS1 expression led to the inhibition of I $\mathrm{B} \alpha \alpha$ phosphorylation and degradation and subsequently to a reduction in NF- $\mathrm{B}$ nuclear translocation. Expression analysis has indicated that the OPN is decreased when BRMS1 is overexpressed in MDA-MB-435 cells; interestingly, a mechanism by which BRMS1 reduces OPN expression levels is via abrogation of NF- $\kappa \mathrm{B}$ activation (8). Another study revealed that BRMS1 expression stimulated p65 dissociation from the $\mathrm{NF}-\kappa \mathrm{B}$ binding site of the uPA promoter, which resulted in reduced transactivation of uPA expression (9). Moreover, BRMS1 has been shown to negatively regulate melanoma angiogenesis by suppressing NF- $\kappa \mathrm{B}$ activity and IL-6 expression (11). Perhaps most interestingly, BRMS1 was shown to reduce CXCR4 expression via abrogation of $\mathrm{NF}-\kappa \mathrm{B}$ signaling, which led to metastasis suppression in lung cancer cells (12). CXCR4 is a seven-domain transmembrane chemokine receptor that is predominantly expressed on lymphocytes where it activates chemotaxis. SDF-1 is the only physiological ligand for CXCR4. The SDF-1/CXCR4 axis has been recently shown to be involved in stimulating multiple metastatic processes in many different neoplasms, including migration, invasion, angiogenesis and proliferation (13-16). Chu et al (17) also demonstrated that CXCR4 overexpression increased vascularity, which may help promote human basal cell carcinoma metastasis. Conversely, both knockdown of CXCR4 and use of a neutralizing antibody against CXCR4 in ovarian carcinoma decreased invasion (28). Moreover, a prior report showed that $\mathrm{NF}-\mathrm{\kappa B}$ could promote migration and organ-specific homing of cancer cells through the induction of CXCR4. The NF- $\mathrm{NB}$ binding site has also been identified in the proximal region of the CXCR4 promoter and is postulated to play a role in CXCR4 expression in human breast cancer cells $(19,28,29)$. Because both BRMS1 and CXCR4 are involved in regulating the $N F-\kappa B$ signaling pathway, we hypothesized that BRMS1 might modulate metastasis of ovarian cancer cells in part by regulating CXCR4 expression. Our data suggested that inhibiting BRMS1 in OVCAR3 cells could lead to the upregulation of CXCR4. We further investigated whether the increase in CXCR4 expression resulting from BRMS1 silencing was due to activation of the NF- $\mathrm{B}$ pathway. To address this question we used an EMSA targeting $\mathrm{NF}-\kappa \mathrm{B}$ binding in the CXCR4 promoter. We determined that blocking BRMS1 obviously increased NF- $\mathrm{KB}$ binding to the CXCR4 promoter compared to the parental cells, whereas an unlabeled competitive sequence markedly inhibited this binding. Taken together, these data provided mechanistic support for our hypothesis that BRMS1 regulates CXCR4 expression through the NF- $\mathrm{NB}$ pathway.

In summary, we report that knockdown of BRMS1 in ovarian cancer cells is associated with upregulation of
CXCR4 mediated by NF- $\kappa$ B activation, which then increases the metastatic potential. Our results contribute to the better understanding of the tumor-suppressive functions of BRMS1 in ovarian cancer and suggest that BRMS1 restoration may be a promising approach for anti-metastasis therapy for human ovarian cancer.

\section{Acknowledgements}

This study was supported by grants from the Natural Science Foundation of Guangdong Province (no. 2009B060700080) and the Science and Information Technology of Guangzhou (no. 2010GN-E00221).

\section{References}

1. Moss $\mathrm{C}$ and Kaye SB: Ovarian cancer: progress and continuing controversies in management. Eur J Cancer 38: 1701-1707, 2002.

2. Seraj MJ, Samant RS, Verderame MF and Welch DR: Functional evidence for a novel human breast carcinoma metastasis suppressor, BRMS1, encoded at chromosome 11q13. Cancer Res 60: 2764-2769, 2000.

3. Shevde LA, Samant RS, Goldberg SF, Sikaneta T, Alessandrini A, Donahue HJ, Mauger DT and Welch DR: Suppression of human melanoma metastasis by the metastasis suppressor gene, BRMS1. Exp Cell Res 273: 229-239, 2002.

4. Zhang S, Lin QD and Di W: Suppression of human ovarian carcinoma metastasis by the metastasis-suppressor gene, BRMS1. Int J Gynecol Cancer 16: 522-531, 2006.

5. Chen X, Xu Z and Wang Y: Recent advances in breast cancer metastasis suppressor 1. Int J Biol Markers 26: 1-8, 2011.

6. DeWald DB, Torabinejad J, Samant RS, Johnston D, Erin N, Shope JC, Xie Y and Welch DR: Metastasis suppression by breast cancer metastasis suppressor 1 involves reduction of phosphoinositide signaling in MDA-MB-435 breast carcinoma cells. Cancer Res 65: 713-717, 2005.

7. Cicek M, Fukuyama R, Welch DR, Sizemore N and Casey G: Breast cancer metastasis suppressor 1 inhibits gene expression by targeting nuclear factor-kappaB activity. Cancer Res 65 : 3586-3595, 2005

8. Samant RS, Clark DW, Fillmore RA, Cicek M, Metge BJ Chandramouli KH, Chambers AF, Casey G, Welch DR and Shevde LA: Breast cancer metastasis suppressor 1 (BRMS1) inhibits osteopontin transcription by abrogating NF- $\kappa \mathrm{B}$ activation. Mol Cancer 6: 6, 2007.

9. Cicek M, Fukuyama R, Cicek MS, Sizemore S, Welch DR, Sizemore N and Casey G: BRMS1 contributes to the negative regulation of uPA gene expression through recruitment of $\mathrm{HDAC} 1$ to the NF- $\kappa \mathrm{B}$ binding site of the uPA promoter. Clin Exp Metastasis 26: 229-237, 2009.

10. Hurst DR, Edmonds MD, Scott GK, Benz CC, Vaidya KS and Welch DR: Breast cancer metastasis suppressor 1 up-regulates miR-146, which suppresses breast cancer metastasis. Cancer Res 69: $1279-1283,2009$

11. Li J, Cheng Y, Tai D, Martinka M, Welch DR and Li G: Prognostic significance of BRMS1 expression in human melanoma and its role in tumor angiogenesis. Oncogene 30: 896-906, 2011.

12. Yang J, Zhang B, Lin Y, Yang Y, Liu X and Lu F: Breast cancer metastasis suppressor 1 inhibits SDF-1 $\alpha$-induced migration of non-small cell lung cancer by decreasing CXCR4 expression. Cancer Lett 269: 46-56, 2008.

13. Kruizinga RC, Bestebroer J, Berghuis P, de Haas CJ, Links TP, de Vries EG and Walenkamp AM: Role of chemokines and their receptors in cancer. Curr Pharm Des 15: 3396-3416, 2009.

14. Manu KA, Shanmugam MK, Rajendran P, Li F, Ramachandran L, Hay HS, Kannaiyan R, Swamy SN, Vail S, Kapoor S, Ramesh B, Bist P, Koay ES, Lim LH, Ahn KS, Kumar AP and Sethi G: Plumbagin inhibits invasion and migration of breast and gastric cancer cells by downregulating the expression of chemokine receptor CXCR4. Mol Cancer 10: 107, 2011.

15. Zhao BC, Wang ZJ, Mao WZ, Ma HC, Han JG, Zhao B and $\mathrm{Xu}$ HM: CXCR4/SDF-1 axis is involved in lymph node metastasis of gastric carcinoma. World J Gastroenterol 17: 2389-2396, 2011 
16. Kajiyama H, Shibata K, Terauchi M, Ino K, Nawa A and Kikkawa F: Involvement of SDF-1 $\alpha / C X C R 4$ axis in the enhanced peritoneal metastasis of epithelial ovarian carcinoma. Int J Cancer 122: 91-99, 2008.

17. Chu CY, Cha ST, Lin WC, Lu PH, Tan CT, Chang CC, Lin BR, Jee SH and Kuo ML: Stromal cell-derived factor-1 $\alpha$ (SDF-1 $\alpha /$ CXCL12)-enhanced angiogenesis of human basal cell carcinoma cells involves ERK1/2-NF- $\mathrm{KB} /$ interleukin-6 pathway. Carcinogenesis 30: 205-213, 2009.

18. Ping YF, Yao XH, Jiang JY, Zhao LT, Yu SC, Jiang T, Lin MC, Chen JH, Wang B, Zhang R, Cui YH, Qian C, Wang J and Bian XW: The chemokine CXCL12 and its receptor CXCR4 promote glioma stem cell-mediated VEGF production and tumour angiogenesis via PI3K/AKT signaling. J Pathol 224: 344-354, 2011.

19. Helbig G, Christopherson KW II, Bhat-Nakshatri P, Kumar S, Kishimoto H, Miller KD, Broxmeyer HE and Nakshatri $\mathrm{H}$ : $\mathrm{NF}-\kappa \mathrm{B}$ promotes breast cancer cell migration and metastasis by inducing the expression of the chemokine receptor CXCR4. J Bilo Chem 278: 21631-21638, 2003.

20. Chambers AF, Groom AC and MacDonald IC: Dissemination and growth of cancer cells in metastatic sites. Nat Rev Cancer 2: 563-572, 2002.

21. Hedley BD, Vaidya KS, Phadke P, Mackenzie L, Dales DW, Postenka CO, MacDonald IC and Chambers AF: BRMS1 suppresses breast cancer metastasis in multiple experimental models of metastasis by reducing solitary cell survival and inhibiting growth initiation. Clin Exp Metastasis 25: 727-740, 2008.

22. Phadke PA, Vaidya KS, Nash KT, Hurst DR and Welch DR BRMS1 suppresses breast cancer experimental metastasis to multiple organs by inhibiting several steps of the metastatic process. Am J Pathol 172: 809-817, 2008.

23. Stark AM, Tongers K, Maass N, Mehdorn HM and Held-Feindt J: Reduced metastasis-suppressor gene mRNA-expression in breast cancer brain metastases. J Cancer Res Clin Oncol 131: 191-198, 2005 .
24. Hicks DG, Yoder BJ, Short S, Tarr S, Prescott N, Crowe JP, Dawson AE, Budd GT, Sizemore S, Cicek M, Choueiri TK, Tubbs RR, Gaile D, Nowak N, Accavitti-Loper MA, Frost AR, Welch DR and Casey G: Loss of breast cancer metastasis suppressor 1 protein expression predicts reduced disease-free survival in subsets of breast cancer patients. Clin Cancer Res 12: 6702-6708, 2006.

25. Zhao XL and Wang P: Expression of SATB1 and BRMS1 in ovarian serous adenocarcinoma and its relationship with clinicopathological features. Sichuan Da Xue Xue Bao Yi Xue Ban 42: 82-85, 105, 2011 (In Chinese).

26. Smith PW, Liu Y, Siefert SA, Moskaluk CA, Petroni GR and Jones DR: Breast cancer metastasis suppressor 1 (BRMS1) suppresses metastasis and correlates with improved patient survival in non-small cell lung cancer. Cancer Lett 276: 196-203, 2009.

27. Zhang Z, Yamashita H, Toyama T, Yamamoto Y, Kawasoe T and Iwase H: Reduced expression of the breast cancer metastasis suppressor $1 \mathrm{mRNA}$ is correlated with poor progress in breast cancer. Clin Cancer Res 12: 6410-6414, 2006.

28. Miyanishi N, Suzuki Y, Simizu S, Kuwabara Y, Banno K and Umezawa K: Involvement of autocrine CXCL12/CXCR4 system in the regulation of ovarian carcinoma cell invasion. Biochem Biophys Res Commun 403: 154-159, 2010.

29. Chua AW, Hay HS, Rajendran P, Shanmugam MK, Li F, Bist P, Koay ES, Lim LH, Kumar AP and Sethi G: Butein downregulates chemokine receptor CXCR4 expression and function through suppression of NF- $\kappa \mathrm{B}$ activation in breast and pancreatic tumor cells. Biochem Pharmacol 80: 1553-1562, 2010. 